\title{
Análise epidemiológica dos óbitos por Doença Hepática Alcoólica entre 2006 e 2015 no Rio Grande do Sul
}

\author{
Epidemiological analysis of Alcoholic Hepatic Disease deaths between 2006 and 2015 in Rio Grande \\ do Sul
}

Análisis epidemiológico de muertes por Enfermedad Hepática Alcohólica entre 2006 y 2015 en Rio

Grande do Sul

Recebido: 14/05/2021 | Revisado: 19/05/2021 | Aceito: 23/05/2021 | Publicado: 09/06/2021

Tomás Zanetti Milani

ORCID: https://orcid.org/0000-0001-5589-5559 Universidade de Passo Fundo, Brasil E-mail: 174424@upf.br

Joao Vitor Barcellos Zin

ORCID: https://orcid.org/0000-0001-6304-1959 Universidade de Passo Fundo, Brasil E-mail: 174407@upf.br

Fernanda Werlang Bamberg ORCID: https://orcid.org/0000-0002-5739-9935 Universidade de Passo Fundo, Brasil E-mail: 168598@upf.br

Leonardo Sérgio Chiodi Mroginski ORCID: https://orcid.org/0000-0003-1836-1931 Universidade de Passo Fundo, Brasil E-mail: 170427@upf.br

Nathalia Beck Corrêa ORCID: https://orcid.org/0000-0001-7531-968X Universidade de Passo Fundo, Brasil E-mail: 175552@upf.br

Vitória Razera Bordignon ORCID: https://orcid.org/0000-0002-5731-5359 Universidade de Passo Fundo, Brasil E-mail: 175642@upf.br

Gabriel Tarasconi Zanin ORCID: https://orcid.org/0000-0002-5082-5364 Universidade de Passo Fundo, Brasil E-mail: 171376@upf.br

Heloisa Todeschini Francescon ORCID: https://orcid.org/0000-0002-0135-3233 Universidade de Passo Fundo, Brasil E-mail: helooisaf_@hotmail.com

Paulo Roberto Reichert ORCID: https://orcid.org/0000-0002-2909-7593 Universidade de Passo Fundo, Brasil E-mail: paulor@upf.br

\begin{abstract}
Resumo
Objetivo: A pesquisa visa descrever o perfil epidemiológico dos óbitos causados pela DHA entre os anos de 2006 e 2015 no estado do Rio Grande do Sul (RS). Método: Por meio do banco de dados do DATASUS e dados demográficos da população de cada região, calculou-se a taxa de mortalidade a cada 100.000 habitantes. A análise foi dividida em sexo (feminino e masculino), ano (2006 a 2015), faixa etária (0-19, 20-59 e 60+) e macrorregiões do RS (Vales, Sul, Serra, Norte, Missioneira, Metropolitana e Centro-Oeste). Resultados: A região Metropolitana apresentou o maior número total de mortes (2277 homens e 279 mulheres), seguida por Norte, Serra, Centro Oeste e Missioneira Sul. Ao analisar o número de mortes por 100.000 habitantes, a região Norte apresentou a maior taxa de mortalidade. Em relação aos sexos, houve maior prevalência do sexo masculino com diferença significativa (p0,012) para as duas variáveis e, no que tange a faixa etária, as maiores taxas foram encontradas no grupo dos idosos (60 anos ou mais). Conclusão: A DHA ainda é uma condição com grande impacto no sistema público de saúde brasileiro. Estratégias para melhorar o rastreamento, políticas públicas voltadas à prevenção do consumo de bebidas alcoólicas e o
\end{abstract}


conhecimento epidemiológico da doença são indispensáveis para haver redução da morbimortalidade tanto no RS quanto em outros estados brasileiros. Incluir o resumo.

Palavras-chave: Doença hepática alcoólica (DHA); Rio Grande do Sul (RS); Mortalidade.

\begin{abstract}
Objective: The research aims to describe the epidemiological profile of deaths due to ALD between the years 2006 and 2015 in the state of Rio Grande do Sul (RS). Methods: Analysis of DATASUS database and demographic population of each region. The mortality rate was calculated for every 100,000 inhabitants and the analysis was divided into sex (female and male), year (2006 to 2015), age group (0-19, 20-59 and 60+) and macro-regions of RS (Valleys, South, Serra, North, Missionary, Metropolitan and Midwest). Results: The Metropolitan region had the highest total number of deaths (2277 men and 279 women), followed by North, Serra, Midwest and Missioneira Sul. Analyzing the number of deaths per 100,000 inhabitants, the North region had the highest mortality rate. Regarding the sexes, there was a higher prevalence of males with significant difference (p0.012) for the two variables and, regarding the age group, the highest rates were found in the elderly group (60 years or older). Conclusion: ALD remains a condition with a major impact on Brazilian's public health system. Strategies to improve screening, public policies aimed to prevent the consumption of alcoholic beverages and the epidemiological knowledge of the disease are essential to reduce morbimortality in RS and others brazilian's states. Incluir o resumo em inglês.
\end{abstract}

Keywords: Alcohol liver disease (ALD); Rio Grande do Sul (RS); Mortality.

\begin{abstract}
Resumen
Objetivo: La investigación tiene como objetivo describir el perfil epidemiológico de las muertes por EHA entre los años 2006 y 2015 en el estado de Rio Grande do Sul (RS). Métodos: Por medio del análisis de la base de datos DATASUS y de la población demográfica de cada región. Se calculó la tasa de mortalidad por cada 100.000 habitantes y el análisis se dividió en sexo (femenino y masculino), año (2006 a 2015), grupo de edad (0-19, 20-59 y $60+)$ y macrorregiones de RS (Valles, Sur, Serra, Norte, Misionero, Metropolitano y Medio Oeste). Resultados: La Región Metropolitana tuvo el mayor número total de muertes (2277 hombres y 279 mujeres), seguida por Norte, Serra, Medio Oeste y Missioneira Sul. Analizando el número de muertes por cada 100.000 habitantes, la región Norte tuvo la tasa de mortalidad más alta. En cuanto a los sexos, hubo mayor prevalencia de varones con diferencia significativa $(\mathrm{p} 0,012)$ para las dos variables $\mathrm{y}$, en cuanto al grupo de edad, las mayores tasas se encontraron en el grupo de ancianos (60 años o más). Conclusión: La EHA sigue siendo una afección con un gran impacto en el sistema público de salud brasileño. Las estrategias para mejorar el rastreamento, las políticas públicas orientadas a prevenir el consumo de bebidas alcohólicas y el conocimiento epidemiológico de la enfermedad son fundamentales para reducir la morbimortalidade em el RS y em los otros estados brasileños. Incluir o resumo em espanhol.
\end{abstract}

Palabras clave: Enfermedad hepática alcohólica (EHA); Rio Grande do Sul (RS); Mortalidad.

\title{
1. Introdução
}

A doença hepática alcoólica (DHA) representa um espectro de lesões hepáticas resultante do consumo crônico de álcool, variando desde a esteatose hepática até formas mais avançadas, incluindo hepatite alcoólica, cirrose e insuficiência hepática crônica (Crabb et al., 2019) (Degré et al., 2020) (Shah et al., 2019). A DHA é uma das principais causas de doença crônica do fígado e é responsável por até $48 \%$ das mortes relacionadas à cirrose nos Estados Unidos. O álcool também é um frequente cofator na aceleração da fibrose em pacientes com outros tipos de doença hepática, como infecção pelo vírus da hepatite C (Singal et al., 2018) (Hagström et al., 2018). Pacientes que consomem 30 gramas ou mais de álcool por dia têm risco aumentado para cirrose, embora a maioria desses indivíduos não desenvolva a doença.

A esteatose é vista em aproximadamente $90 \%$ dos etilistas e, desses, um terço desenvolverá esteatohepatite se permanecer em consumo de bebidas alcoólicas (Guirguis et al., 2015). Para tanto, a investigação é realizada em pacientes com história compatível, transaminases séricas elevadas (ALT/AST), imagens sugestivas de esteatose hepática ou esteatose encontrada em uma biópsia hepática. O diagnóstico é estabelecido após afastar-se outras causas de esteatose hepática ou de cirrose, como vírus da hepatite, obesidade ou doenças genéticas. (Guirguis et al., 2015) Como a abstinência alcoólica é o único e mais importante fator para a melhora da sobrevida desses pacientes (Crabb et al., 2019), o tratamento multidisciplinar com especialistas é primordial e qualquer forma de deteç̧ão de áreas com maiores taxas de alcoolismo podem aumentar a acessibilidade a esses recursos.

Além do aconselhamento sobre a dependência alcoólica, a farmacoterapia para DHA muitas vezes pode ser uma 
possibilidade. Entretanto, fármacos como o dissulfiram e a naltrexona - muito comuns no histórico de tratamento da doença exibem hepatotoxicidade significativa, limitando seu uso em doenças hepáticas avançadas e encontrando-se em desuso atualmente. Isso remete ao fato da importância do trabalho psicossocial desses pacientes, seja juntamente a grupos de apoio ou por meio de especialistas na área de terapia cognitivo-comportamental. (Rachakonda et al., 2020).

A análise de dados epidemiológicos relacionados ao etilismo é escassa a nível estadual, ainda mais quando relacionado à mortalidade. Em virtude da importância do debate frente ao consumo de bebidas alcoólicas nas diversas esferas sociais, seja para diferentes classes, idades ou até gêneros, qualquer impacto causado por dados do assunto pode fortalecer pautas de saúde e prevenção. Dessa forma, o estudo tem como objetivo descrever o perfil epidemiológico dos óbitos por DHA no estado do Rio Grande do Sul (RS) entre os anos de 2006 e 2015, haja visto a grande repercussão do tema em nosso meio.

\section{Metodologia}

A presente pesquisa trata-se de um estudo descritivo e retrospectivo (Pereira et al, 2018) (Ludke. \& Andre, 2013), com objetivo de traçar o perfil epidemiológico das mortes notificadas por doença alcoólica hepática (CID10- K70) no período entre janeiro de 2006 e dezembro de 2015 no estado do RS. Tais dados foram obtidos por meio do Departamento de Informática do Sistema Único de Saúde do Brasil - DATASUS, via ferramenta TABNET.

A coleta de dados foi realizada por meio da busca do número absoluto de mortes notificadas pela doença alcoólica do fígado, estratificando-as por faixa etária (0-9 anos;10-19 anos; 20-29 anos; 30-39 anos; 40-49 anos; 50-59 anos; 60-69 anos; 70-79 anos e 80 anos ou mais), período (2006-2015) e macrorregiões de saúde do RS (Vales, Sul, Serra, Norte, Missioneira, Metropolitana e Centro-Oeste). Ademais, buscou-se a população total de cada região estudada também pela plataforma DATASUS. Para quantificar a taxa de mortalidade a cada 100.000 habitantes, usou-se o seguinte cálculo: número de óbitos multiplicado por 100.000 e dividido pelo número de habitantes em cada localidade.

Após serem obtidos, os dados foram tabulados e armazenados no programa Microsoft Excel. A análise estatística foi feita por meio do programa SPSS (versão 16.0, livre comercialmente) e de testes estatísticos não paramétricos (Friedmann \& Wilcoxon) para amostras dependentes, já que a população doente estudada continuava na amostra com o passar dos anos. Para a realização deste estudo não foi necessária a aprovação pelo Comitê de Ética, visto que se trata de uma análise baseada em um banco de dados público.

\section{Resultados}

Ao analisar o número de mortes absolutas por meio do CID K70, totalizaram-se 5589 mortes no Estado do RS. A macrorregião que obteve o maior número de mortes foi a região Metropolitana com 2556 mortes (2277 homens e 279 mulheres), seguida por Norte com 850 mortes (762 homens e 88 mulheres), Serra com 757 mortes (680 homens e 77 mulheres), Centro Oeste com 556 mortes (510 homens e 46 mulheres), Missioneira com 465 mortes (426 homens e 39 mulheres) e Sul com 405 mortes (362 homens e 43 mulheres).

Comparando as 7 macrorregiões em saúde no período selecionado por óbitos a cada 100 mil habitantes, a região Norte obteve a maior taxa de mortalidade com 6,8742 mortes por 100.000 habitantes, seguido pela região Serra com 6,8185, Vales com 6,1529, a região Metropolitana com 5,4608, região Centro Oeste 5,4472, região Missioneira 5,1317 e Sul 3,8942. Ao comparar todas as Macrorregiões a cada ano por meio do teste não paramétrico de Friedmann, houve diferença estatisticamente significativa $(\mathrm{p}=0,423)$. O Gráfico 1 demonstra a evolução da mortalidade por região neste período. 
Gráfico 1 - Evolução da taxa de mortalidade por 100.000 habitantes nas macrorregiões do Rio Grande do Sul no período de 2006 a 2015.

\section{Evolução da taxa de mortalidade por região}

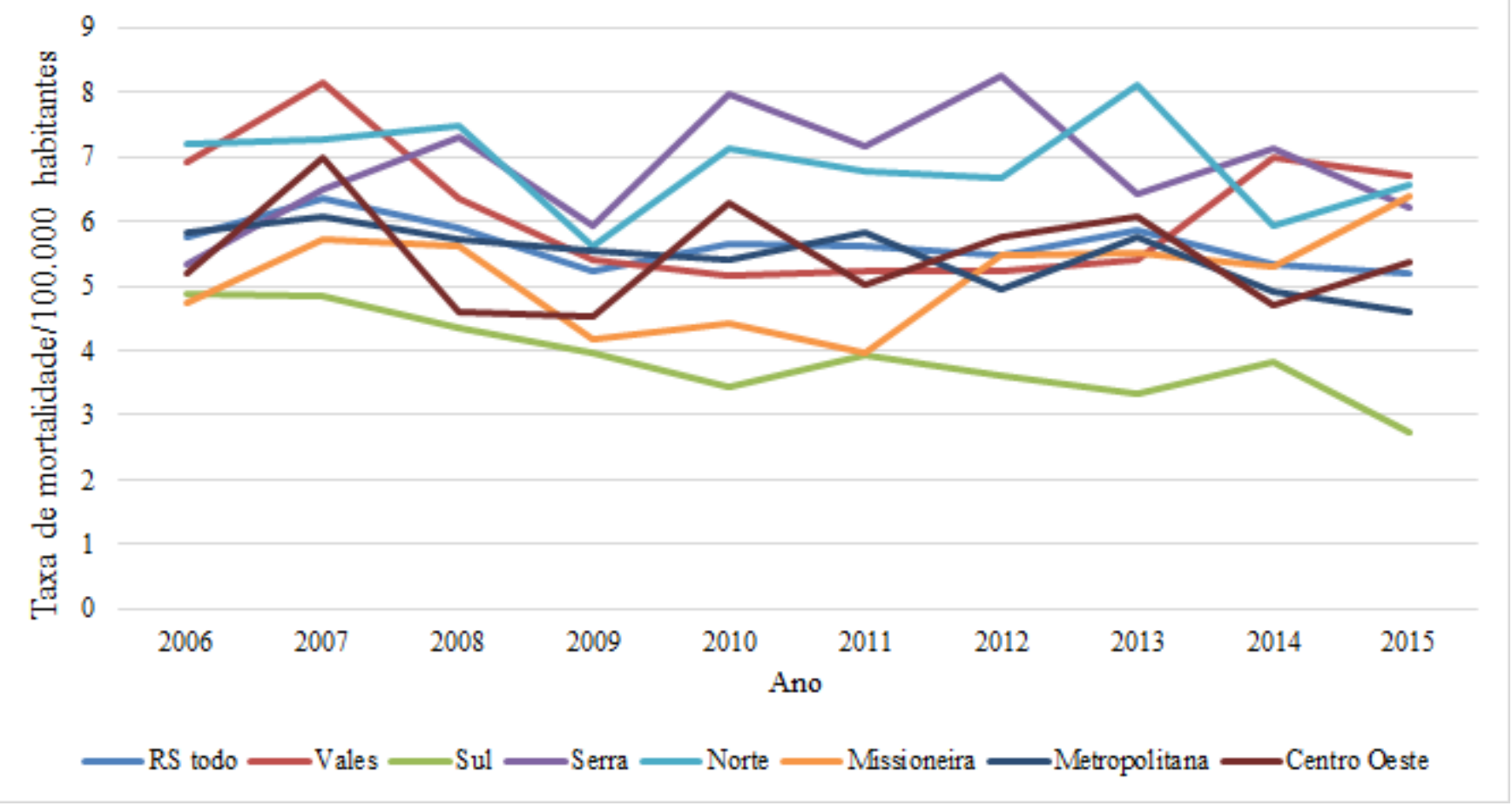

[Observa-se que as regiões da Serra, Missioneira e Centro Oeste apresentaram variação positiva na taxa de mortalidade ao longo do período, destacando-se a região Missioneira, a qual obteve uma variação positiva de 1,673. Já as regiões Vales, Sul, Norte e Metropolitana variaram negativamente a taxa de mortalidade, merecendo ênfase a região Sul, com decréscimo de 2,117]

Fonte: elaborado pelos autores.

Nos anos de análise houveram 5503 mortes masculinas e 625 femininas devido a doença. A média do número absoluto de óbitos das sete regiões avaliadas, no período, para o sexo masculino foi de 1375,63 ( $(1780,04$; IC95\%= $-112,53-$ $2863,78)$ e para o sexo feminino $156,25( \pm 205,33 ;$ IC $95 \%=-15,41-327,91)$. A diferença entre as médias foi de 1219,38 . Já a média da taxa de mortalidade por 100 mil habitantes das sete regiões avaliadas para o sexo masculino foi de 10,2584 $( \pm 1,7213$; IC95\%=8,8194-11,6975) e para o feminino $1,0921( \pm 0,2375$; IC95\%=0,8936-1,2907). A diferença entre as médias foi de 9,1663. Ao compararmos as duas médias entre os sexos, tanto em número absoluto quanto em taxa de mortalidade por 100 mil habitantes, pelo teste de Wilcoxon, a diferença foi significativa $(\mathrm{p}=0,012)$. O Gráfico 2 demonstra a distribuição da taxa de mortalidade por gênero nas regiões estudadas. 
Gráfico 2 - Distribuição média da taxa de mortalidade por gênero e macrorregião durante o período analisado (20062015).

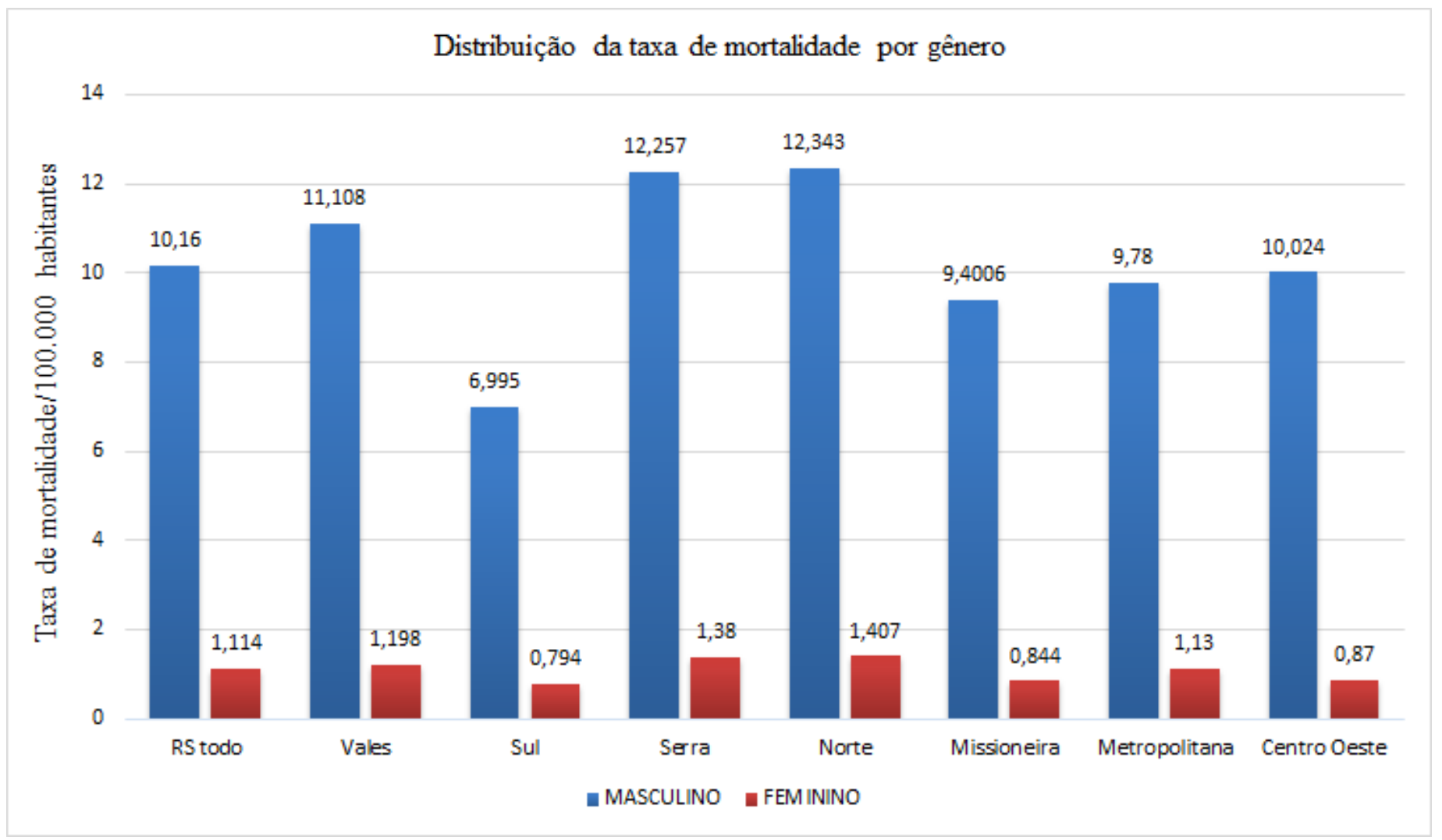

[Percebe-se que a taxa de mortalidade/100.000 habitantes é consideravelmente maior no sexo masculino, apresentando uma variação média de 2,96, enquanto no sexo feminino esta variação é de 0,056 . Além disso, observa-se que a menor taxa de mortalidade no sexo masculino foi de 6,995 (região Sul), que quando comparada com a maior taxa do sexo feminino, 1,407 (região Norte), essa é quase 5 vezes maior] Fonte: elaborado pelos autores.

Como apresentado no Gráfico 3, ao analisar a taxa de mortalidade a cada 100 mil habitantes do RS por faixa etária, os resultados obtidos foram 0,0091 para 0-19 anos, 6,5552 para 20-59 anos e 13,5470 para 60 anos ou mais. Ao comparar as faixas etárias entre as Macrorregiões, as maiores taxas foram encontradas no grupo dos idosos (60 anos ou mais) em todas as Macrorregiões, com diferença significativa encontrada por meio do teste não paramétrico de Friedmann, com $\mathrm{p} \leq 0,001$. 
Gráfico 3 - Distribuição da taxa de mortalidade/100.000 habitantes por faixa etária e macrorregião no período analisado (2006-2015).

\section{Distribuição da taxa de mortalidade/100.000 habitantes por faixa etária e macrorregião}

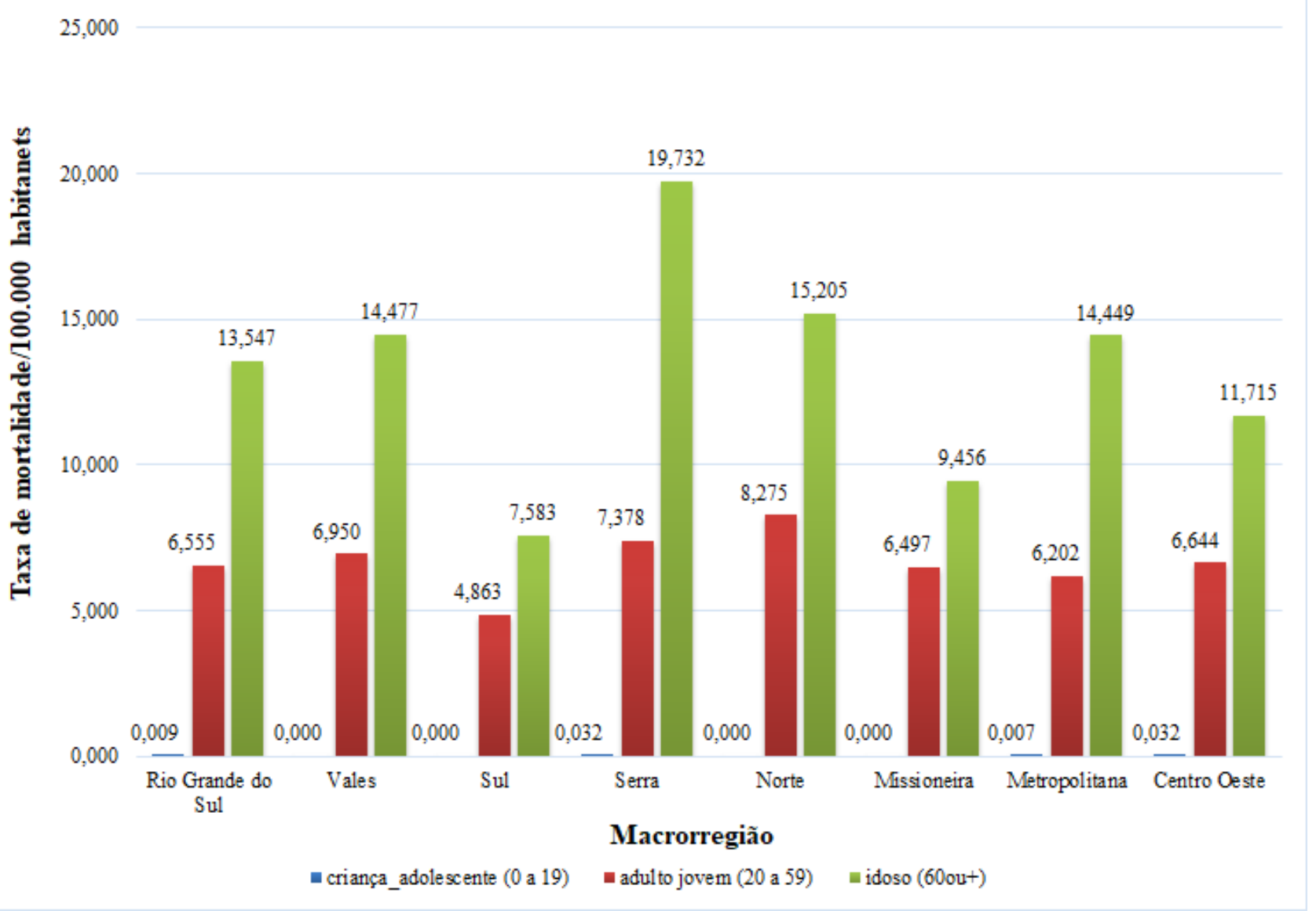

[Nota-se que a representatividade da taxa de mortalidade/100.000 habitantes em crianças e adolescentes é extremamente baixa, até mesmo sendo nula em algumas macrorregiões, como Vales, Sul, Norte e Missioneira. Quando analisada a faixa etária com mais de 60 anos, percebese uma discrepância com relação às demais, sendo maior em todas as macrorregiões. Já os adultos jovens apresentam uma taxa de mortalidade intermediária dentre as analisadas]

Fonte: elaborado pelos autores.

\section{Discussão}

O presente estudo avaliou as características sociodemográficas das mortes por DHA no RS, identificadas no DataSUS, o maior banco de dados de saúde do Brasil. Com base nos resultados, evidenciou-se uma considerável mortalidade por DHA no Estado do RS, no período de 2006-2015. Com relação aos números absolutos, a região Metropolitana se destaca. Ao analisar o número de mortes por 100.000 habitantes, a região Norte apresentou a maior taxa de mortalidade, seguida da região da Serra e dos Vales. Destaca-se que o consumo de bebidas alcoólicas em locais de temperaturas mais baixas parece ser superior do que em locais mais quentes e isso poderia contribuir para o maior número de mortes por habitantes encontradas nessas macrorregiões do RS (Ventura-Cots et al., 2019).

A região Sul do Brasil, composta pelos estados de Santa Catarina, Paraná e Rio Grande do Sul, a partir de uma análise nacional feita por Lyra, A.C. e colaboradores, mostrou que o número de mortes absolutas por DHA de 2006 a 2015 totalizaram 17.036. Ao estudo, o RS apresentou cerca de 33\% das mortes por DHA da região Sul e a taxa de mortalidade por 1000 habitantes aumentou nesse período. Somado a isso, a maior taxa de mortalidade do Brasil ocorreu em 2011 na região Sul, com 
6.49 a cada 1000 habitantes - somente em 2013 e 2015 a região Sul não apresentou a maior taxa de mortalidade por 1000 habitantes do Brasil. Analisando a proporção de mortes do RS com outros estados da região Sul, o Estado colaborou, portanto, com a taxa de mortalidade elevada apresentada. (Lyra, 2020)

O espectro da DHA varia desde esteatose, esteatohepatite e até cirrose, que é a maior precursora do carcinoma hepatocelular (CHC) (Naveau et al., 1997) (Crabb, 1999). O CHC é, muitas vezes, diagnosticado tardiamente, pois apresentase de forma silenciosa e pouco sintomática (Atkins et al., 2014) (Kansagara et al., 2014) (Ganne-Carrié \& Nahon, 2019). O prognóstico dos pacientes com esse tumor apresenta curso desfavorável, com uma taxa de sobrevivência em cinco anos de $18 \%$ (Villanueva, 2019) (Mahmud et al., 2020). Um estudo publicado em 2020, que buscou analisar os dados epidemiológicos de CHC no Brasil, evidenciou que a região Sul apresentou o maior número relativo de casos de $\mathrm{CHC}$ ( $>20$ casos / 100.000 habitantes) em comparação aos outros estados (Fernandes et al., 2020). Além disso, o estudo demonstrou que a maior porcentagem dos casos de CHC tinha uma média de idade de 60 anos e que os casos eram mais frequentes no sexo masculino (Fernandes et al., 2020).

O consumo alcoólico é significativamente maior pelo sexo masculino do que pelo feminino, cujos dados encontrados mostram que a média da taxa de mortalidade por 100.000 habitantes das sete regiões avaliadas para o sexo masculino foi de $10,2584( \pm 1,7213$; IC95\%=8,8194-11,6975) e para o feminino de $1,0921( \pm 0,2375$; IC95\%=0,8936-1,2907) com diferença significativa de 9,1663. Dados semelhantes, em proporções gerais, mostram que os homens apresentam mais hospitalizações do que as mulheres em todos estados brasileiros ( $81.68 \%$ vs $18.32 \%)$ e, também, maiores taxas de mortalidade, variando de 86.22\% ( $\mathrm{n}=6293)$ na região centro-oeste para 89.70\% ( $=15282)$ na região Sul do Brasil. (Lyra, 2020).

A respeito da faixa etária, houve alguma discrepância ao analisarmos pontualmente a faixa de idade com maior número de mortes comparando com o estudo acima, o qual mostrou que as maiores mortes e hospitalizações na região Sul do Brasil ocorreram entre indivíduos de 50-59 anos (Lyra, 2020), enquanto a análise do RS mostrou que o maior número de óbitos ocorreu naqueles com 60 anos ou mais.

É importantíssimo salientar que a Classificação Internacional de Doenças (CID) K70 pode não abranger a totalidade de mortes por cirrose hepática alcoólica. Isso se deve a uma parcela das mortes por cirrose, independente da causa, estarem vinculadas ao CID K74 - Fibrose e cirrose hepáticas. Apesar da abreviação do CID K70 especificar em 70.3 "Cirrose hepática alcoólica", muitos centros anexam apenas a causa base do óbito, que é cirrose. Dessa forma, os números encontrados podem estar subestimados, entretanto as relações feitas permanecem fidedignas.

O presente estudo apresenta algumas limitações, pois foi realizado a partir das informações do DataSUS, que abrange apenas pacientes da rede pública de saúde. Ademais, temos acesso apenas ao número bruto de mortes, de idade e de gênero, sem ter acesso ao prontuário, não tendo como avaliar quais foram as evoluções e as intercorrências dos pacientes. Outro ponto importante a ser observado é que os dados obtidos são referentes ao número de mortes e não ao número de casos existentes de DHA durante o período estudado. Por essas razões, os resultados do estudo devem ser interpretados com cautela. Apesar das limitações descritas, os resultados encontrados refletem a mortalidade na saúde pública de um país em desenvolvimento frente à uma doença comum e que, na sociedade atual, apresenta subsídios para continuar se desenvolvendo. Sendo assim, a utilização dessa base de dados tem a vantagem de uma análise ampla com um maior número de pacientes, permitindo a geração de evidências relevantes para a tomada de decisão e condutas quando se trata de políticas públicas em saúde.

\section{Considerações Finais}

A DHA ainda é uma condição com grande impacto no sistema público de saúde brasileiro devido às diversas complicações associadas, sendo subdiagnosticada ou diagnosticada já em estágios avançados com danos irreversíveis ao fígado. Estratégias para melhorar o rastreamento de pacientes com DHA visando o diagnóstico precoce e evitando a progressão 
da doença são indispensáveis para a redução na morbimortalidade. Além disso, políticas públicas voltadas à prevenção do consumo de bebidas alcoólicas é mandatório, pois a doença é totalmente prevenível.

Em suma, enfatiza-se a importância de dar continuidade para as pesquisas de cunho epidemiológico a respeito da DHA e sua mortalidade. Recomendamos a análise ampla da CID, sempre tentando abranger as causas de morte a serem estudadas em mais que uma codificação, visando diminuir qualquer viés. Por fim, encorajamos futuros pesquisadores a valorizar qualquer dado geográfico de óbitos por DHA, bem como por outras doenças, de maneira a concentrar medidas direcionadas à saúde da população.

\section{Referências}

Atkins, D., Ross, D., \& Kelley, M. (2014). Acting in the Face of Uncertainty. Annals of Internal Medicine, 161(4), 300. https://doi.org/10.7326/m14-1344.

Crabb, D. W. (1999). Pathogenesis of Alcoholic Liver Disease. Newer Mechanisms of Injury. The Keio Journal of Medicine, 48(4), 184-188. https://doi.org/10.2302/kjm.48.184.

Crabb, D. W., Im, G. Y., Szabo, G., Mellinger, J. L., \& Lucey, M. R. (2020). Diagnosis and Treatment of Alcohol-Associated Liver Diseases: 2019 Practice Guidance From the American Association for the Study of Liver Diseases. Hepatology, 71(1), 306-333. https://doi.org/10.1002/hep.30866.

Degré, D., Stauber, R. E., Englebert, G., Sarocchi, F., Verset, L., Rainer, F., Spindelboeck, W., Njimi, H., Trépo, E., Gustot, T., Lackner, C., Deltenre, P., \& Moreno, C. (2020). Long-term outcomes in patients with decompensated alcohol-related liver disease, steatohepatitis and Maddrey's discriminant function \&lt;32. Journal of Hepatology, 72(4), 636-642. https://doi.org/10.1016/j.jhep.2019.12.023.

Fernandes, G. dos., Campos, D., Ballalai, A., Palhares, R., Alvares-da-Silva, M. R., Palhares, D. M., Neto, B.-H. F., Barros, F. M., Gil, R. de, Chagas, A., \& Carrilho, F. J. (2020). Author Correction: Epidemiological and Clinical Patterns of Newly Diagnosed Hepatocellular Carcinoma in Brazil: the Need for Liver Disease Screening Programs Based on Real-World Data. Journal of Gastrointestinal Cancer. https://doi.org/10.1007/s12029-020-00539-0.

Ganne-Carrié, N., \& Nahon, P. (2019). Hepatocellular carcinoma in the setting of alcohol-related liver disease. Journal of Hepatology, 70(2), $284-293$. https://doi.org/10.1016/j.jhep.2018.10.008.

Guirguis, J., Chhatwal, J., Dasarathy, J., Rivas, J., McMichael, D., Nagy, L. E., McCullough, A. J., \& Dasarathy, S. (2015). Clinical Impact of AlcoholRelated Cirrhosis in the Next Decade: Estimates Based on Current Epidemiological Trends in the United States. Alcoholism: Clinical and Experimental Research, 39(11), 2085-2094. https://doi.org/10.1111/acer.12887.

Hagström, H., Hemmingsson, T., Discacciati, A., \& Andreasson, A. (2018). Alcohol consumption in late adolescence is associated with an increased risk of severe liver disease later in life. Journal of Hepatology, 68(3), 505-510. https://doi.org/10.1016/j.jhep.2017.11.019.

Kansagara, D., Papak, J., Pasha, A. S., O’Neil, M., Freeman, M., Relevo, R., Quiñones, A., Motu'apuaka, M., \& Jou, J. H. (2014). Screening for Hepatocellular Carcinoma in Chronic Liver Disease. Annals of Internal Medicine, 161(4), 261. https://doi.org/10.7326/m14-0558.

Ludke, M., \& Andre, M. E. D. A. (2013). Pesquisas em educação: uma abordagem qualitativa: E.P.U. F.

Lyra, A. C., Almeida, L. M., Mise, Y. F., \& Cavalcante, L. N. (2020). Epidemiological profile of alcoholic liver disease hospital admissions in a Latin American country over a 10-year period. World Journal of Hepatology, 12(5), 230-238. https://doi.org/10.4254/wjh.v12.i5.230.

Mahmud, N., Fricker, Z., Hubbard, R. A., Ioannou, G. N., Lewis, J. D., Taddei, T. H., Rothstein, K. D., Serper, M., Goldberg, D. S., \& Kaplan, D. E. (2020). Risk Prediction Models for Post-Operative Mortality in Patients With Cirrhosis. Hepatology, 73(1), 204-218. https://doi.org/10.1002/hep.31558.

Naveau, S., Giraud, V., Borotto, E., Aubert, A., Capron, F., \& Chaput, J. (1997). Excess weight risk factor for alcoholic liver disease. Hepatology, 25(1), 108111. https://doi.org/10.1002/hep.510250120.

Pereira, A. S, Shitsuka D. M., Parreira F. J., Shitsuka R. (2018). Metodologia da pesquisa científica. [e-book]. Santa Maria. Ed.UAB/NTE/UFSM. https://repositorio.ufsm.br/bitstream/handle/1/15824/Lic_Computacao_Metodologia-Pesquisa-Cientifica.pdf?sequence=1. Acesso em: 25 jan. 2021.

Rachakonda, V., Bataller, R., \& Duarte-Rojo, A. (2020). Recent advances in alcoholic hepatitis. F1000Research, 9, 97. https://doi.org/10.12688/f1000research.20394.1.

Shah, N. D., Ventura-Cots, M., Abraldes, J. G., Alboraie, M., Alfadhli, A., Argemi, J., Badia-Aranda, E., Arús-Soler, E., Barritt, A. S., Bessone, F., Biryukova, M., Carrilho, F. J., Fernández, M. C., Dorta Guiridi, Z., El Kassas, M., Eng-Kiong, T., Queiroz Farias, A., George, J., Gui, W., \& Bataller, R. (2019). Alcohol-Related Liver Disease Is Rarely Detected at Early Stages Compared With Liver Diseases of Other Etiologies Worldwide. Clinical Gastroenterology and Hepatology, 17(11). https://doi.org/10.1016/j.cgh.2019.01.026.

Singal, A. K., Bataller, R., Ahn, J., Kamath, P. S., \& Shah, V. H. (2018). ACG Clinical Guideline: Alcoholic Liver Disease. American Journal of Gastroenterology, 113(2), 175-194. https://doi.org/10.1038/ajg.2017.469.

Stein, E., Cruz-Lemini, M., Altamirano, J., Ndugga, N., Couper, D., Abraldes, J. G., \& Bataller, R. (2016). Heavy daily alcohol intake at the population level predicts the weight of alcohol in cirrhosis burden worldwide. Journal of Hepatology, 65(5), 998-1005. https://doi.org/10.1016/j.jhep.2016.06.018. 
Research, Society and Development, v. 10, n. 6, e54010616105, 2021

(CC BY 4.0) | ISSN 2525-3409 | DOI: http://dx.doi.org/10.33448/rsd-v10i6.16105

Ventura-Cots, M., Watts, A. E., Cruz-Lemini, M., Shah, N. D., Ndugga, N., McCann, P., Barritt, A. S., Jain, A., Ravi, S., Fernandez-Carrillo, C., Abraldes, J. G., Altamirano, J., \& Bataller, R. (2019). Colder Weather and Fewer Sunlight Hours Increase Alcohol Consumption and Alcoholic Cirrhosis Worldwide. Hepatology, 69(5), 1916-1930. https://doi.org/10.1002/hep.30315.

Villanueva, A. (2019). Hepatocellular Carcinoma. New England Journal of Medicine, 380(15), 1450-1462. https://doi.org/10.1056/nejmra1713263. 